\title{
1 Ascorbate maintains a low plasma low oxygen level
}

\section{Authors}

4 Louise Injarabian ${ }^{1,2}$, Marc Scherlinger ${ }^{3}$, Anne Devin², Stéphane Ransac², Jens $5 \quad$ Lykkesfeldt $^{4}$, Benoit S Marteyn ${ }^{1,5,6^{*}}$

\section{Affiliations}

$8{ }^{1}$ Université de Strasbourg, CNRS, Architecture et Réactivité de l'ARN, UPR9002, F967000 Strasbourg, France

10 2Université de Bordeaux, IBGC, UMR 5095, 1 rue Camille Saint Saëns, 33077

11 Bordeaux Cedex, France

12 3UMR-CNRS UMR -5164 Immunoconcept, 146 rue Léon Saignat 33076 Bordeaux,

13 France

$14{ }^{4}$ Faculty of Health and Medical Sciences, University Copenhagen

15 Institut Pasteur, Unité de Pathogenèse des Infections Vasculaires, 28 rue du Dr Roux, 1675724 Paris Cedex 15, France

17 6INSERM Unité 1225, 28 rue du Dr Roux, 75724 Paris Cedex 15, France 18

19 *Corresponding author: Benoit Marteyn (marteyn@unistra.fr), Institut de Biologie 20 Moléculaire et Cellulaire Pasteur, 15, rue Descartes 67000 Strasbourg France; Phone $21+33388417083$.

22 
1 Key points

2

3 Abstract

4 In human blood, oxygen is mainly transported by red blood cells. Accordingly,

5 the oxygen level in plasma is expected to be limited, although it has not been

6 quantified yet. Here, by developing dedicated methods and tools, we determined

7 that human plasma $\mathrm{pO}_{2}=8.4 \mathrm{mmHg}\left(1.2 \% \mathrm{O}_{2}\right)$. Oxygen solubility in plasma was

8 believed to be similar to water. Here we reveal that plasma has an additional ascorbate-dependent oxygen-reduction activity. Plasma oxygenation oxidizes ascorbate $(49.5 \mu \mathrm{M}$ in fresh plasma vs $<2 \mu \mathrm{M}$ in oxidized plasma) and abolishes this capacity, which is restored by ascorbate supplementation. We confirmed these results in vivo, showing that the plasma $\mathrm{pO}_{2}$ is significantly higher in ascorbate-deficient guinea pigs (plasma ascorbate $<2 \mu \mathrm{M}$ ), compared to control

14 (plasma ascorbate $>15 \mu \mathrm{M}$ ). Plasma low oxygen level preserves the integrity of oxidation-sensitive components such as ubiquinol. Circulating leucocytes are well adapted to these conditions, since the abundance of their mitochondrial

17 network is limited.

18 These results shed a new light on the importance of oxygen exposure on 19 leucocyte biological study, in regards with the reducing conditions they 20 encounter in vivo; but also on the manipulation of blood products to improve 21 their integrity and potentially improve transfusions' efficacy. 


\section{Introduction}

2 Blood plasma is composed of water, ions, organic molecules, such as proteins, and

3 vitamins. Gases, such as carbon dioxide $\left(\mathrm{CO}_{2}\right)$ and oxygen $\left(\mathrm{O}_{2}\right)$ also enter into the

4 plasma composition, according to Henry's law. The solubility coefficient of $\mathrm{O}_{2}\left(\alpha_{\mathrm{O} 2}\right)$ in

5 plasma at $37^{\circ} \mathrm{C}$ is low compared to the $\mathrm{CO}_{2}\left(\alpha_{\mathrm{O} 2}=0.0031 \mathrm{~mL} \mathrm{O} / \mathrm{mmHg} / 100 \mathrm{~mL}\right.$ blood

6 vs $\alpha_{\mathrm{cO} 2}=0.069 \mathrm{~mL} \mathrm{CO} / \mathrm{mmHg} / 100 \mathrm{~mL}$ blood) ${ }^{1}$. Only a limited fraction of $\mathrm{O}_{2}$ is

7 dissolved in plasma, representing less than $2 \%$ of the total blood oxygen content.

8 Arterial $\mathrm{pO}_{2}$ equals $75-100 \mathrm{mmHg}$ and venous $\mathrm{pO}_{2}$ equals $30-50 \mathrm{mmHg}$; in theory the

9 blood plasma $\mathrm{pO}_{2}$ would be ranged from 0.9 to $3 \mathrm{mmHg}$. The plasma fraction is

10 anticipated to be poorly oxygenated in the overall blood circulation, although it has not

11 been experimentally quantified. Until now, it was considered that the solubility

12 coefficient of $\mathrm{O}_{2}$ in plasma was similar in water or saline ${ }^{2}$. The impact of ascorbate (or

13 Vitamin C), a strong reducing molecule, on plasma oxygen level has not yet been

14 investigated, despite of its abundance (plasma ascorbate concentration $50-70 \mu \mathrm{M}^{3,4}$ )

15 and the respective standard redox potential of $\mathrm{O}_{2}$ and ascorbate $\left(\mathrm{E}^{\prime}{ }^{0} \mathrm{O} / \mathrm{H} 2 \mathrm{O}=0.815\right.$ and

$16 E^{\prime}{ }_{D H A} /$ Ascorbate $=0.08$ at $\left.25^{\circ} \mathrm{C}, \mathrm{P}=1 \mathrm{~atm}, \mathrm{pH}=7\right)^{5}$.

17 In this report, by developing innovative strategies and tools we assessed plasma

oxygen level and stability for the first time. We confirmed experimentally that plasma

is poorly oxygenated and revealed that ascorbate contributes to its low oxygenation level, by reducing $\mathrm{O}_{2}$. The impact of plasma "physiological hypoxia" on circulating cells' physiology and components' stability and redox status has been further investigated.

22

\section{Methods}


1 Blood samples were collected either in commercial collection tubes (BD Vacutainer

2 K2E (EDTA), ref 368861) or in Hypoxytubes developed in collaboration with the

3 Greiner Bio One (GBO) company, containing a limited amount of $\mathrm{O}_{2}$. Internal $\mathrm{pO}_{2}$ was

4 quantified in commercial tubes and in Hypoxytubes using an oximeter with a

5 microsensor equipped with a steel needle (Unisense).

6

7 Blood collection

8 All participants gave written informed consent in accordance with the Declaration of

9 Helsinki principles. Human blood was collected from healthy patients at the ICAReB

10 service of the Pasteur Institut (authorization No. 2020_0120).

Cell culture

HEK293T (ATCC CRL-1573) and Hep-G2 (ATCC HB-8065) were cultured in DMEM +

$8 \%$ SVF. Cells were seeded onto 24 -well plates and incubated $24 \mathrm{~h}$ at $37^{\circ} \mathrm{C}$ at $0 \%$ (anoxic cabinet) or $21 \% \mathrm{O}_{2}$.

16 White blood cells (WBCs) were purified form whole blood in an anoxic chamber by the 17 addition of a $6 \%$ dextran solution (30 min, RT). The WBC-containing supernatant was collected and resuspended in RPMI 1640 (Thermofisher); remaining red blood cells were eliminated with a lysis buffer.

20 Cells were fixed in paraformaldehyde (PFA) $3.3 \%$ for immunofluorescent labelling or

21 labeled with fluorescent marker for flow cytometry analysis, as previously described 6 . 
1 Immediately after blood collection, the plasma $\mathrm{pO}_{2}$ was measured directly in the blood

2 collection tube using an oximeter with a standardized microsensor equipped with a

3 steel needle (Unisense), as previously described ${ }^{7}$.

4

5 Following centrifugation for $5 \mathrm{~min}$ at 2,000 $\times \mathrm{g}$, the plasma was acidified with an equal

6 volume of $10 \%(\mathrm{w} / \mathrm{v})$ metaphosphoric acid (MPA) containing $2 \mathrm{mmol} / \mathrm{L}$ of disodium-

7 EDTA. Ascorbate concentration was quantified by high-performance liquid

8 chromatography with coulometric detection, as described previously ${ }^{8}$. Likewise, using

9 high-performance liquid chromatography with coulometric detection, $\alpha$ - and $\gamma$ -

10 tocopherol were analyzed as described by Sattler et al. ${ }^{9}$, and ubiquinone and ubiquinol

11 as described elsewhere ${ }^{10}$.

12 Plasma potassium, calcium, magnesium, albumin, fibrinogen, Factor V and Factor VIII

13 were quantified by a medical laboratory (Cerballiance, Paris, France).

Plasma oxygen reduction rate quantification

16 Plasma oxygen consumption rate was measured with an oxymeter (Oroboros O2k17 FluoRespirometer). Immediately after blood collection, samples were centrifuged, and 18 plasma fractions were loaded in closed cuves $(2 \mathrm{~mL})$. Oxygen consumption fluxes were 19 assessed when reaching constant values. Experiments were conducted with fresh 20 plasma and after oxidation (exposure to atmospheric air: at least 30 min on a rotator 21 mixer).

Mitochondria study

24 Imaging. Mitochondria were immunolabeled with anti-SDHA antibody (ab14715, 25 Abcam) in combination with a conjugated Alexa Fluor-568 (2124366, Invitrogen) on 
1 fixed preparations. Nuclei were labeled with DAPI. Cell imaging was performed with a

2 confocal microscope (Leica DM5500 TCS SPE).

3 Flow cytometry. Cells were resuspended in PBS + 2 mM EDTA, labeled with $100 \mathrm{nM}$

4 TMRM (T5428, Sigma-Aldrich) and analyzed with FACSCcalibur (BD Biosciences).

5 Data were quantified with the FlowJo software (FlowJo, LLC).

6

$7 \quad$ Guinea pig plasma analysis $\pm 2.9 \mathrm{mmHg}$ ) hereafter termed Hypoxytube (Figure 1A), to avoid experimental oxygenation of blood samples. Oxygen level in plasma was quantified with a needle

3-week Dunkin-Hartley guinea pigs (Charles River) were fed for fifteen days with a standard diet (400 mg ascorbate/kg, Safediet ref. 106) or an ascorbate-deficient diet (<50 mg ascorbate/kg). Blood samples were collected in Hypoxytubes; plasma ascorbate concentration and $\mathrm{pO}_{2}$ were determined as described above. Procedure approved by the Institut Pasteur ethics committee (auth. $\mathrm{n}^{\circ} 190127$ ).

\section{Statistics}

Data were analyzed with the Prism 8 software (GraphPad). ANOVA or Student T-test were performed to analyze the different datasets.

\section{Results and Discussion}

\section{Blood plasma is poorly oxygenated}

Since all commercial tubes contain a significant amount of oxygen (here, $75.7 \pm 4.6$ $\mathrm{mmHg}$ ), we designed and produced tubes containing a limited amount of oxygen (15.9 sensor in commercial tubes or Hypoxytubes (Figure 1B). Plasma $\mathrm{pO}_{2}$ was $9.8 \pm 4.8$ 
$1 \mathrm{mmHg}$ in commercial tubes versus $8.4 \pm 1.0 \mathrm{mmHg}$ in Hypoxytube $(p<0.01)$ : the lastest

2 value being the most accurate quantification of the plasma oxygen level so far (Figure 3 1C).

\section{Ascorbate sustains a plasma oxygen-reduction activity}

6 Oxygen solubility has so far been believed to be similar in plasma and water. However,

7 when fresh plasma $\mathrm{pO}_{2}$ was recorded in a closed chamber (Oroboros), a continuous decrease was observed until anoxia was reached (Figure 1D). This reaction was significantly lower in oxidized plasma (exposed to atmospheric oxygen) or in water (Figure 1D-E). These results strongly suggested that an oxygen-sensitive plasma

11 component was mediating its oxygen-reduction capacity. We hypothesized that 12 plasma ascorbate may play a central role in this reaction. The supplementation of oxidized plasma with $200 \mu \mathrm{M}$ ascorbate restored its oxygen reduction activity (Figure 1D and $1 \mathrm{~F})$, supporting this hypothesis. This ascorbate-dependent reaction does not occur in water (Figure S1A). The plasma ascorbate concentration was first determined in a large number of individuals $(49.5 \pm 14.2 \mu \mathrm{M}$, Figure $1 \mathrm{G})$. We further demonstrated that plasma ascorbate concentration was drastically reduced in oxidized plasma compared to fresh plasma $(p<0.001$, Figure $1 \mathrm{H})$, confirming ascorbate susceptibility to oxidation as previously reported. We further demonstrated that the concentration of ubiquinol, another oxidation-sensitive plasma component was significantly lower in

21 oxidized plasma compared to fresh plasma $(p<0.05$, Figure 11$)$; this reaction was 22 associated with an increase of ubiquinone (ubiquinol oxidized form), as expected 23 (Figure 1I). The concentration of other plasma components was not modified by 24 plasma oxygenation, including salts (potassium, calcium, magnesium, Figure S1B), 
1 proteins (albumin, fibrinogen, coagulation Factor V and VIII, Figure S1C) or additional

2 oxidation-sensitive components ( $\alpha$-tocopherol, $\gamma$-tocopherol) (Figure S1D).

3 We confirmed the ascorbate-dependent plasma oxygen reduction capacity in vivo, in

4 a guinea pig model. Like humans, guinea pigs do not synthesize ascorbate, and are

5 consequently dependent on dietary supply. When animals were fed a standard diet

6 (400 mg/kg ascorbate), the plasma ascorbate concentration was higher than $15 \mu \mathrm{M}$

7 and the plasma $\mathrm{pO}_{2}$ controlled at a low level $(24.11 \pm 2.23 \mathrm{mmHg}$, Figure $1 \mathrm{~J}-\mathrm{K})$. These

8 values are comparable to human plasma although higher, probably due to technical

9 reasons (increased lag time between blood collection and $\mathrm{pO}_{2}$ measurement). When

10 animals were fed an ascorbate-deficient diet ( $<50 \mathrm{mg}$ ascorbate $/ \mathrm{kg}$ ), the plasma

11 ascorbate concentration was lower than $2 \mu \mathrm{M}$ and the plasma $\mathrm{pO}_{2}$ no longer

12 maintained at a low level $(50.40 \pm 26.32 \mathrm{mmHg})$ (Figure 1J-K). Altogether these results

13 confirm the in vivo contribution of ascorbate to the maintenance of a low plasma 14 oxygen level.

Circulating leucocytes sense plasma low-oxygenation - mitochondrial network

17 The adaptation of circulating leucocytes to plasma low oxygen level has not previously 18 been investigated. In other cell-types, it has been reported that under hypoxic conditions, mitochondrial abundance and oxygen consumption is reduced ${ }^{11-13}$. In addition, mitochondria play a key function in leucocyte metabolism and function,

21 beyond metabolism regulation ${ }^{14}$. We confirmed by immunofluorescence (Figure $2 \mathrm{~A}$ ) 22 and flow cytometry (Figure 2B-D) that, compared to two different cell lines (HEK293T 23 and HEp-2) cultured under atmospheric conditions, the mitochondrial abundance of 24 leucocytes (granulocytes, monocytes and lymphocytes) was significantly reduced 25 (ANOVA, Figure 2C-D). Cell lines' mitochondrial content was significantly reduced 
1 upon culture under anoxic conditions (Figure S2C). These results confirm that

2 leucocytes evolve under low oxygen conditions in the blood plasma fraction.

3

4 Blood plasma low oxygenation level should be further considered for basic research and therapeutic application

6

7 Blood plasma low oxygen level should be considered more carefully to study blood 8 cells in vitro.

9 It may have some important drawback in blood product collection and preservation prior transfusion to avoid detrimental impact on its quality.

12 Further investigations will be required to determine the intermediate reactions involved 13 in the reduction of $\mathrm{O}_{2}$ by ascorbate in the plasma, potentially involving the transient 14 formation of reactive oxygen species or ascorbate free radical ${ }^{5}$.

16 Plasma transfusion, preservation of samples from oxidation: red blood cells ${ }^{15}$, platelet 1716

Acknowledgements.

This work was supported by the French National Research Agency : ANR JCJC 201717-CE15-0012 (BSM).

22

\section{Authorship contributions}

$24 \mathrm{LI}$ conducted quantitative analysis of the data. MS performed experiments. JL 25 quantified plasma ascorbate in plasma samples. LI, AD and SR contributed to data 
1 interpretation. BSM designed and performed the experiments, interpreted the data,

2 and wrote the manuscript. All authors critically edited the draft manuscript and 3 approved the final version.

4

5 Disclosure of Conflicts of Interest

6 Authors declare no conflict of interest

7

References

11 Figure legends

Figure 1. The plasma oxygen level is low, mainly sustained by the ascorbate oxygen. (A) Blood collection tubes containing a limited amount of oxygen

14 (Hypoxytubes) have been designed and validated using an oximeter with a microsensor equipped with a steel needle. Commercial tube used as a control was BD Vacutainer K2E (EDTA). Results are expressed as Mean \pm S.D.; ${ }^{* * *}$ indicates $p<0.0001, n=20$. (B-C) Plasma oxygen level was directly quantified in whole venous blood collected in commercial tube or Hypoxytube. Plasma $\mathrm{pO}_{2}$ quantifications are expressed as Mean \pm S.D.; ** indicates $p<0.01, n=12$.

(D) Plasma samples were

20 loaded in closed cuve to record the time-dependent oxygen availability in fresh plasma,

21 oxidized plasma supplemented or not with $200 \mu \mathrm{M}$ ascorbate, and water

22 (representative experiment). (E) Plasma oxygen reduction rates were quantified in

23 fresh or oxidized plasma samples, as described in (D). Results are expressed as Mean $24 \pm$ S.D.; * indicates $p<0.05, n=5$. (F) The impact of oxidized plasma ascorbate 
1 supplementation $(200 \mu \mathrm{M})$ was quantified as described in (E). Results are expressed

2 as Mean \pm S.D.; ** indicates $p<0.01, n=4$. (G-H) Plasma ascorbate concentration in

3 fresh samples was quantified as described in Methods, in blood samples collected in

4 Hypoxytubes $(\mathrm{G}, \mathrm{n}=18)$. The impact of plasma oxygenation on ascorbate concentration

5 is shown in $(H, n=4)$. Results are expressed as Mean \pm S.D.; ${ }^{* *}$ indicates $p<0.001$. (I)

6 Ubiquinol concentration in fresh and oxidized plasma samples was quantified, together

7 with other plasma components (see Figure S1). Results are expressed as Mean \pm

8 S.D.; * indicates $\mathrm{p}<0.05, \mathrm{n}=3$. (J-K) The impact of plasma ascorbate deficiency on the

9 control of the oxygen level has been investigated in vivo in guinea pigs (J-K). Plasma

10 ascorbate concentration and $\mathrm{pO} 2$ was recorded in animals fed standard (400 mg

11 ascorbate/kg) or ascorbate-deficient diet (<50 mg ascorbate/kg) (J). Plasma pO2 was

12 average in each group (plasma ascorbate $<2 \mu \mathrm{M}$ (deficient) and $>15 \mu \mathrm{M}$ (control)).

13 Results are expressed as Mean \pm S.D.; ** indicates $p<0.01, n=6$.

14 Figure 2. The mitochondria abundance is reduced in circulating leucocytes in

15 low-oxygenated plasma. (A) Immunofluorescence staining of white blood cells (WBCs : monocytes, lymphocytes and granulocytes) Hep-G2 cells, and HEK293T cells using anti-CII (mitochondria, red) and DAPI (nuclei, blue). Bars are $10 \mu \mathrm{m}$. (B) Flow cytometry analysis of WBCs (granulocytes, monocytes and lymphocytes), Hep-G2 and HEK293T. Representation of SSC and FSC profiles. (C) TMRM (mitochondria) intensity profiles in granulocytes, monocytes, lymphocytes, Hep-G2 and HEK293T

21 cells (representative experiment) (D) Quantification of TMRM mean fluorescence 22 intensity $(\mathrm{MFI})$ in cells described in (C). Results are expressed as Mean \pm S.D.; **** 23 indicates $p<0.0001$ (One-way ANOVA with Tukey's test, see Tables in Figure S1A), $24 \mathrm{n}=4$. See additional quantification in Figure S2C. 
2 Supplementary data. Figure 2.

3 (A) Water oxygen reduction rate w/wo Asc. (B) Plasma component quantification in

4 fresh an oxidized plasma samples

\section{Supplementary data. Figure 2.}

7 (A) Results of ANOVA statistical analysis (Tukey's multiple comparisons test) of TMRM

8 MFI between granulocytes, monocytes, lymphocytes, HepG2 and HEK293T cells

9 (Figure 2D), representing mean difference, 95\% confidence interval of the difference,

10 significance, summary and adjusted p-value. (B) Details of ANOVA statistical analysis

11 test. (C) TMRM MFI in HepG2 and HEK293T cells exposed to atmospheric oxygen

$12\left(21 \%\right.$ O2) or anoxia $\left(0 \%\right.$ O2) for $24 \mathrm{~h}$ at $37^{\circ} \mathrm{C}$ measured with flow cytometry. (D)

13 Representation of TMRM profiles of HepG2 and HEK293T cells exposed or not to 14 atmospheric oxygen (21\%).

\section{References}

17 1. Pittman RN. Regulation of Tissue Oxygenation. Colloquium Ser Integr Syst

Funct.

$2011 ; 3(3): 1-100$.

2. Christmas KM, Bassingthwaighte JB. Equations hfor $\mathrm{O} 2$ and $\mathrm{CO} 2$ Solubilities in

21 Saline and Plasma: Combining Temperature and Density Dependences. J Appl 
2 3. VanderJagt D, Garry P, Hunt W. Ascorbate in plasma as measured by liquid 3 chromatography and by dichlorophenolindophenol colorimetry. Clin Chem. $4 \quad 1986 ; 32(6): 1004-6$.

6 4. Lykkesfeldt J, Tveden-Nyborg P. The Pharmacokinetics of Vitamin C. Nutrients. $7 \quad 2019 ; 11(10): 2412$.

9 5. Cabelli DE, Bielski BH. Kinetics and mechanism for the oxidation of ascorbic acid/ascorbate by $\mathrm{HO} 2 / \mathrm{O} 2-$ (hydroperoxyl/superoxide) radicals. A pulse radiolysis and stopped-flow photolysis study. J Phys Chem. 1983;87(10):1809-1812.

6. Monceaux V, Chiche-Lapierre C, Chaput C, et al. Anoxia and glucose supplementation preserve neutrophil viability and function. Blood. 2016;128(7):9931002.

7. Tinevez J-Y, Arena ET, Anderson M, et al. Shigella-mediated oxygen depletion is essential for intestinal mucosa colonization. Nat Microbiol. 2019;4(11):2001-2009.

8. Lykkesfeldt J. Measurement of ascorbic acid and dehydroascorbic acid in biological samples. Curr Protoc Toxicol Éditor Board Mahin D Maines Ed Et Al. 2002; Chapter

24 9. Sattler W, Mohr D, Stocker R. Rapid isolation of lipoproteins and assessment of their 25 peroxidation by high-performance liquid chromatography postcolumn 
1 chemiluminescence.

Methods

Enzymol.

1994;233:469-89.

2

3 10. Schou-Pedersen AV, Schemeth D, Lykkesfeldt J. Determination of Reduced and

4 Oxidized Coenzyme Q10 in Canine Plasma and Heart Tissue by HPLC-ECD:

5 Comparison with LC-MS/MS Quantification. Antioxidants. 2019;8(8):253.

6

7 11. Fukuda R, Zhang H, Kim J, et al. HIF-1 Regulates Cytochrome Oxidase Subunits

8 to Optimize Efficiency of Respiration in Hypoxic Cells. Cell. 2007;129(1):111-122.

9

12. Zhang H, Gao P, Fukuda R, et al. HIF-1 Inhibits Mitochondrial Biogenesis and

11 Cellular Respiration in VHL-Deficient Renal Cell Carcinoma by Repression of C-MYC

12 Activity.

Cancer

Cell.

2007;11(5):407-420.

13. Papandreou I, Cairns RA, Fontana L, Lim A, Denko NC. HIF-1 mediates adaptation

to hypoxia by actively downregulating mitochondrial oxygen consumption. Cell Metab. 2006;3(3):187-197.

14. Mehta MM, Weinberg SE, Chandel NS. Mitochondrial control of immunity: beyond

ATP.

Nat

Rev

Immunol.

2017;17(10):608-620.

15. Mohanty JG, Nagababu E, Rifkind JM. Red blood cell oxidative stress impairs oxygen delivery and induces red blood cell aging. Front Physiol. 2014;5:84.

24 16. Manasa K, Vani R. Influence of Oxidative Stress on Stored Platelets. Adv 
A
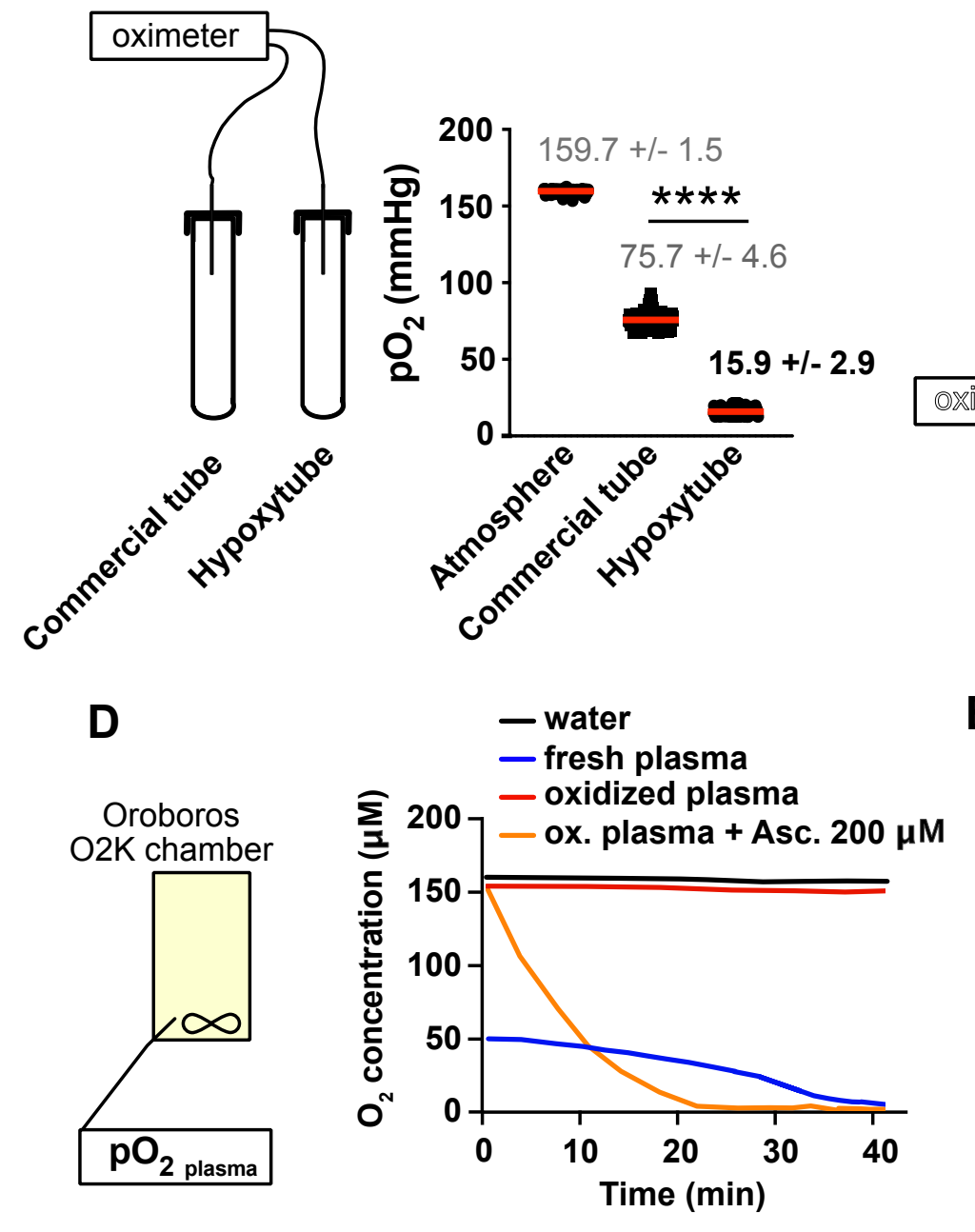

G

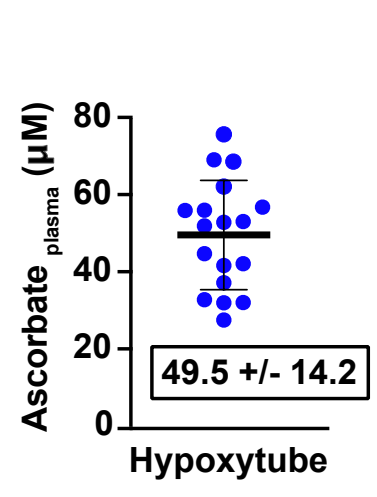

H

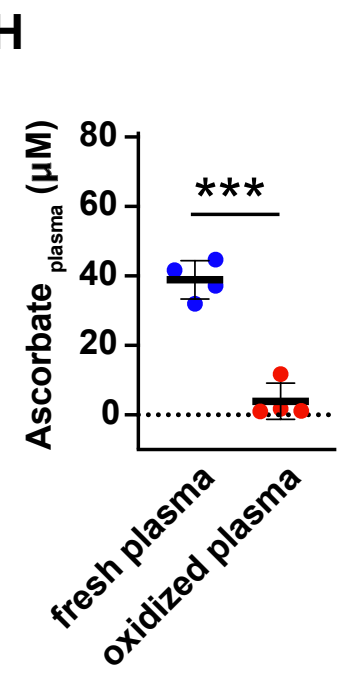

E

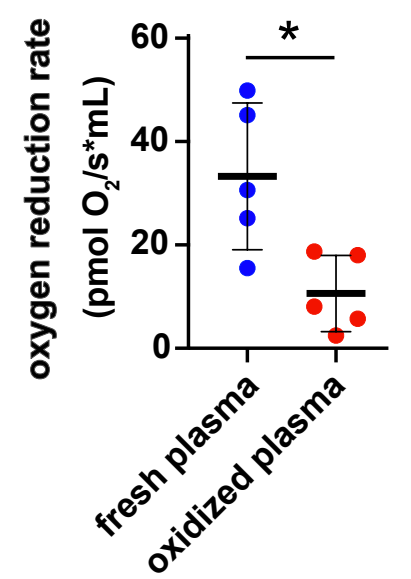

C

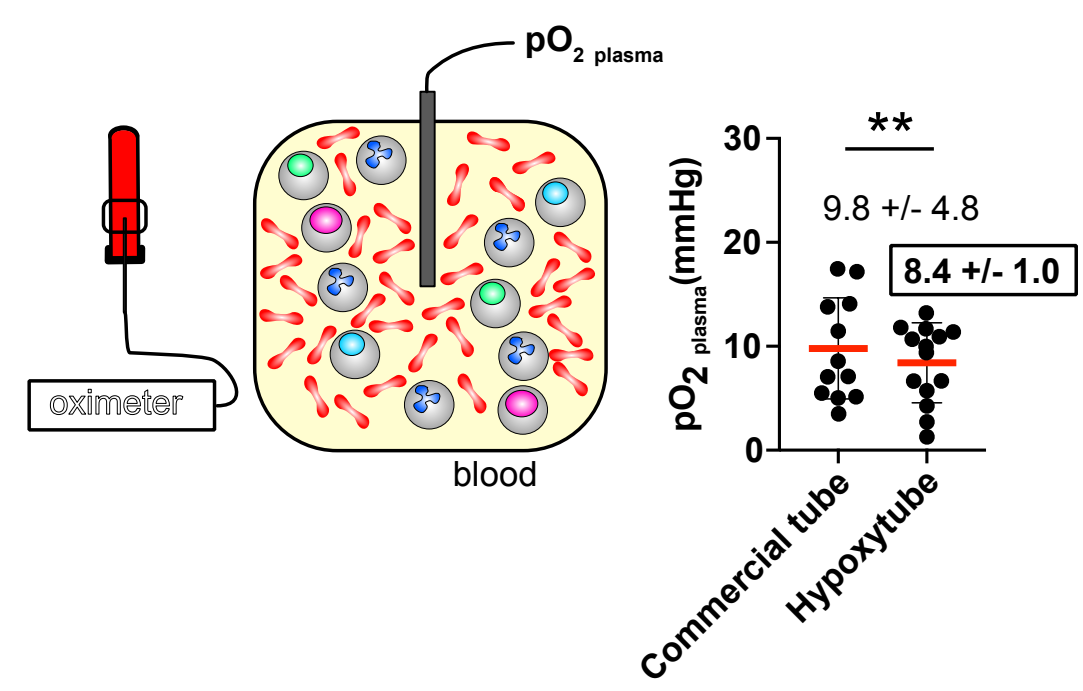

F
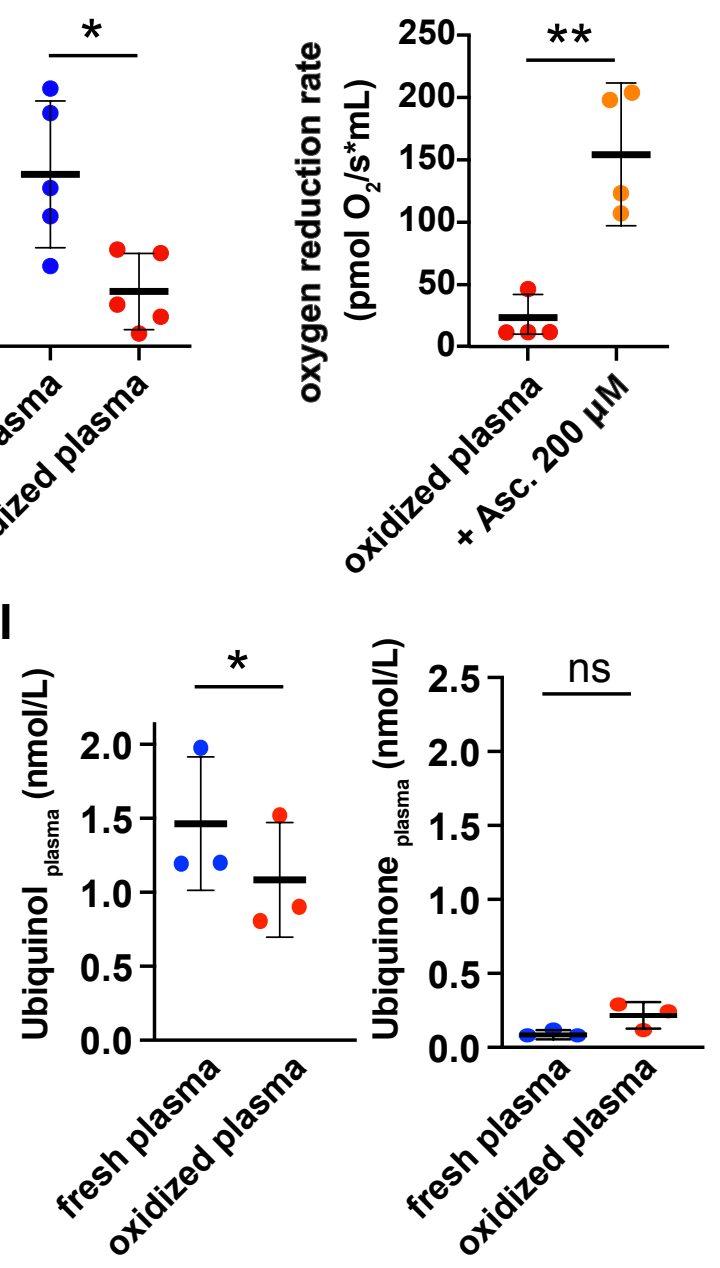

J

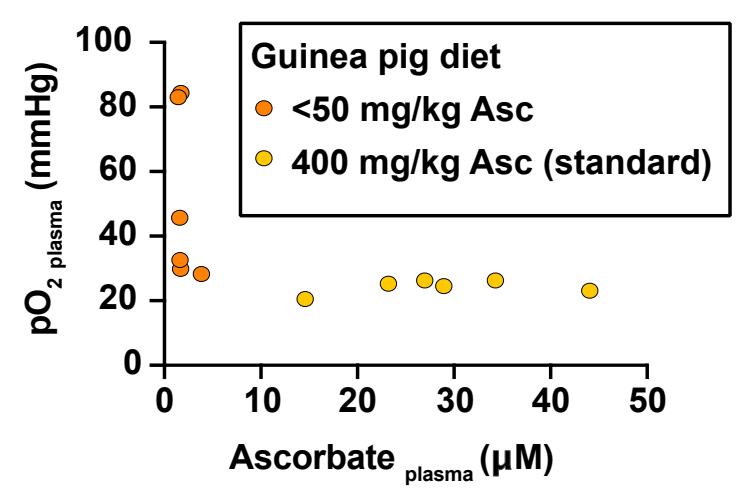

K

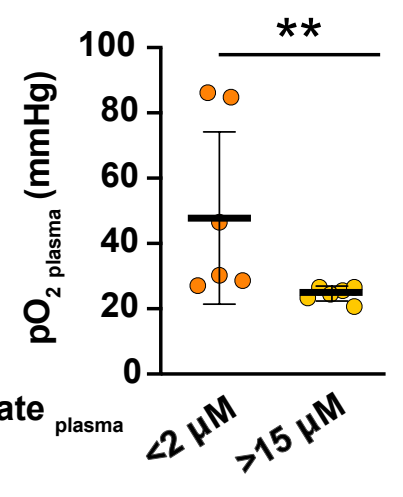


Iniarabi bioRxiv prepript doi;https://doi.grg/10.1101/2020.02.06.937037; this version posted February 7, 2020. The copyright holder for this A

B

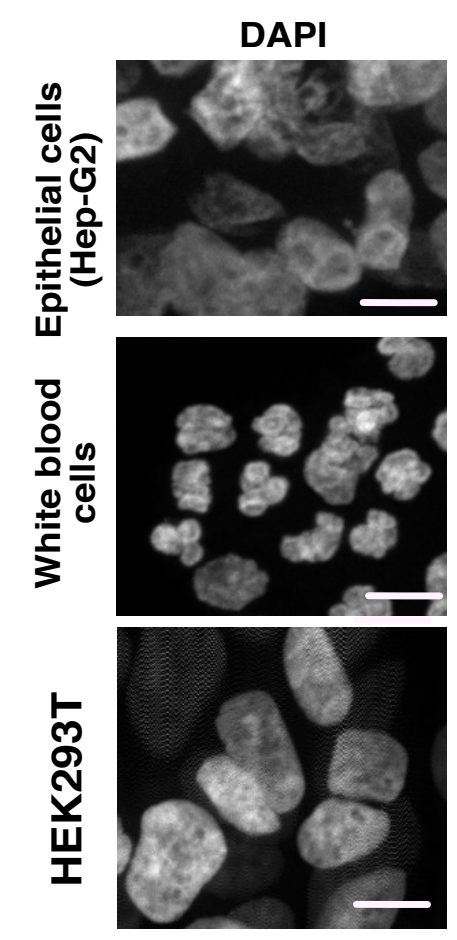

C
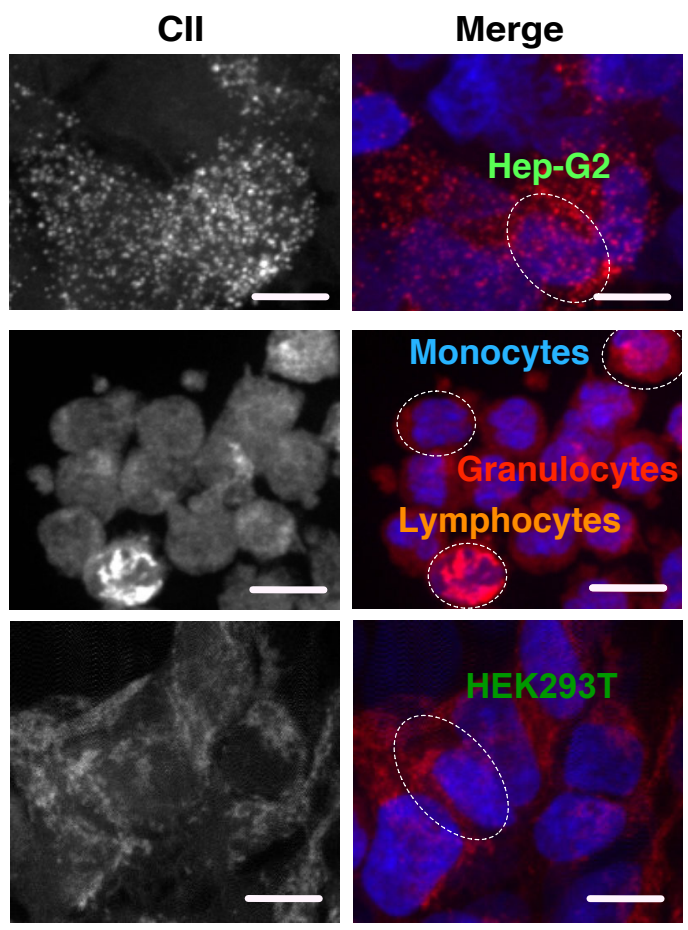

D

Merge
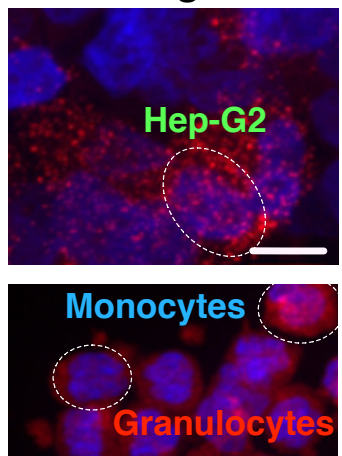

\section{Lymphocytes}

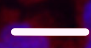

D
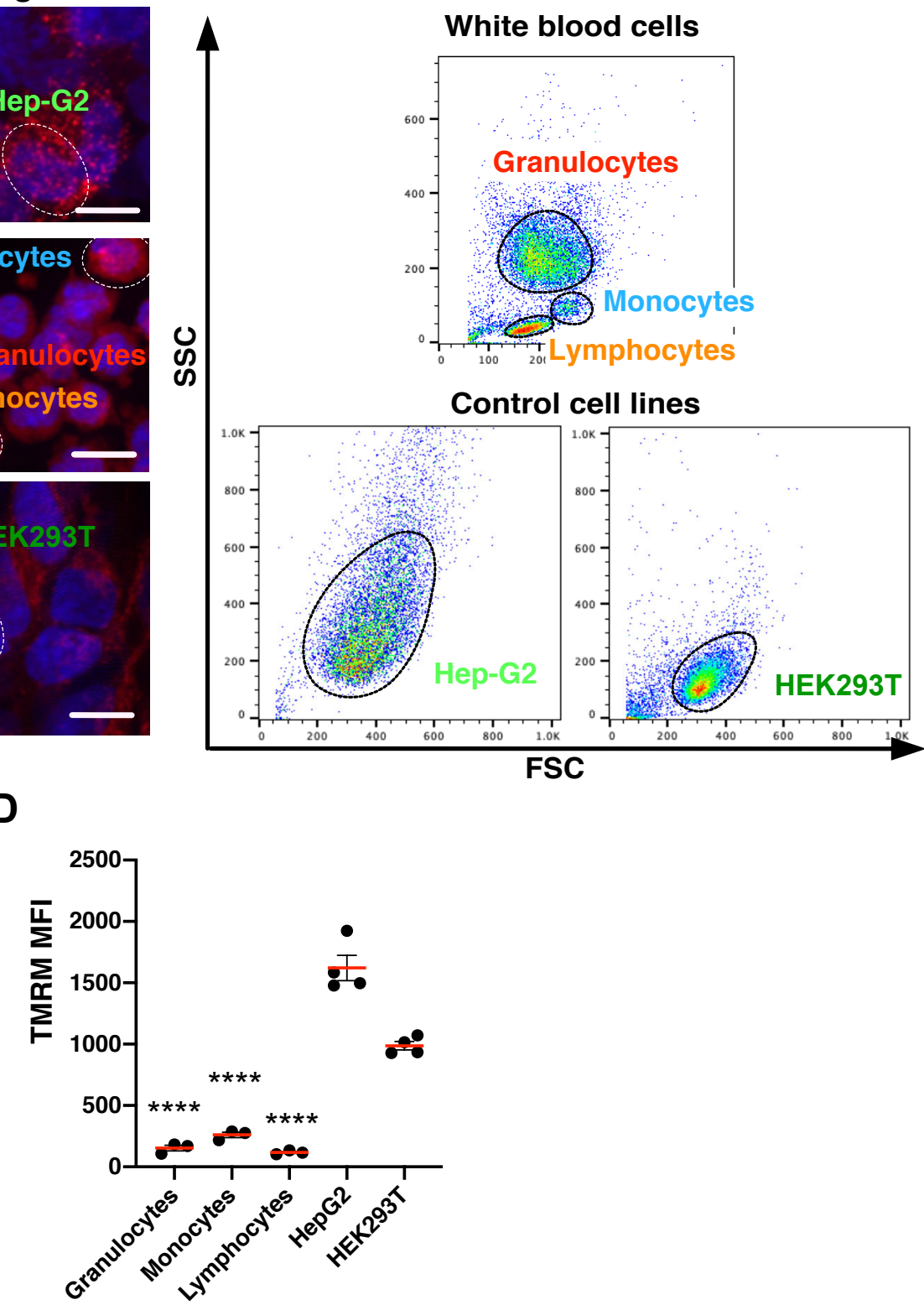
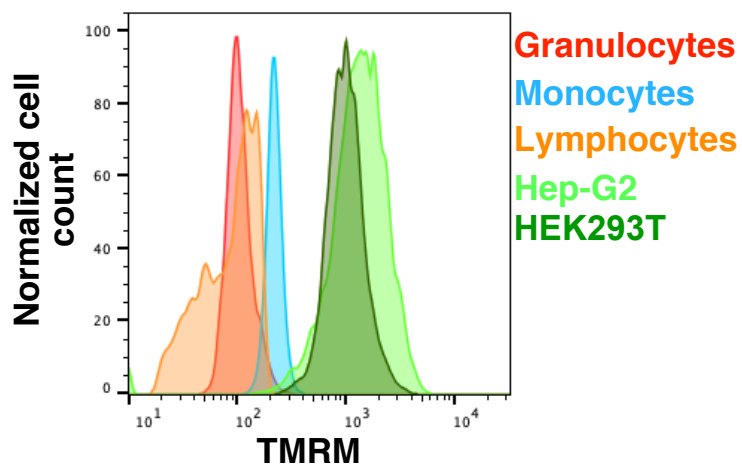